\title{
Three-dimensional modelling of the human carotid artery using the lattice Boltzmann method. I: Model and velocity analysis
}

\author{
J Boyd $\dagger$ and J M Buick $\ddagger$ \\ $\dagger$ Cardiovascular Research Group, Physics, University of New England, Armidale, \\ NSW, 2351, Australia \\ $\ddagger$ Mechanical \& Design Engineering, Anglesea Building, Anglesea Road, University of \\ Portsmouth, Portsmouth, PO1 3DJ UK
}

\begin{abstract}
.
Numerical modelling is a powerful tool in the investigation of human blood flow and arterial diseases such as atherosclerosis. It is known that near wall velocity and shear are important in the pathogenesis and progression of atherosclerosis. In this paper results for a simulation of blood flow in a three-dimensional carotid artery geometry using the lattice Boltzmann method are presented. The velocity fields in the body of the fluid are analysed at six times of interest during a physiologically accurate velocity waveform. It is found that the three-dimensional model agrees well with previous literature results for carotid artery flow. Regions of low near wall velocity and circulatory flow are observed near the outer wall of the bifurcation and in the lower regions of the external carotid artery, which are regions that are typically prone to atherosclerosis.
\end{abstract}

PACS numbers: 47.11.-j, 47.11.Qr, 47.63.-b, 87.19.U-

Submitted to: Phys. Med. Biol.

\section{Introduction}

Cardiovascular disease is one of the principle causes of morbidity in the industrialised world $[1,2,3]$. Pathologies encompassing all vascular sclerosis or pathological abnormalities resulting in vessel wall thickening and hardening of the vessels are designated by the generic term of arteriosclerosis [4]. Of these, atherosclerosis is the principle pathology [2].

The earliest recognizable lesion of atherosclerosis is the "fatty streak", which consists of an aggregation of macrophages and $\mathrm{T}$ lymphocytes in the extracellular matrix just below the endothelial lining of the intima in the larger arteries $[1,5]$. These fatty streaks can then develop into intermediate lesions, composed of a lipid core and layers of macrophages that attract smooth muscle cells from the underlying layer of vascular 
smooth muscle. Intermediate lesions can develop into more advanced and obstructive lesions called fibrous plaques. These plaques protrude into the lumen and can disrupt blood flow, they are covered by a dense calcified cap consisting of fibrous connective tissue with embedded smooth muscle cells that usually overlays a core of lipids and necrotic debris $[1,5]$.

In the advanced stages of the disease, the plaque may occlude the artery to such an extent that oxygen supply to cells can be disrupted, resulting in inefficient cell function and even cell death. Atherosclerosis is a principle contributor to myocardial and cerebral infarction, gangrene and loss of function in the extremities [5]. Heart attacks and strokes can be caused when plaques rupture and release thrombi that block smaller vessels downstream of the plaque, depriving cells of oxygen and leading to their death.

The progression of atherosclerosis is not independent of the flow properties of the blood surrounding it. It is a geometrically focal disease [6] that occurs most frequently in the outer edges of arterial bifurcations [6], and the curved sections of large arteries [7]. These regions are typically areas of disturbed flow patterns $[8,6]$, low velocity and low shear rates $[9,10,11]$. It is not clear why these flow factors influence the progression of atherosclerosis, but it has been postulated $[7,9,10]$ that the disturbed flow zones and low shear stress lead to an increased residency time for particles near the artery wall that can promote or increase plaque progression. Atherosclerosis in the carotid artery tends to be located near the bifurcation, in the sinus portion and in the curved terminal part of the internal carotid artery [12].

The study of the haemodynamic properties of the blood in these regions of the artery can lead to a greater understanding of atherosclerosis and its dependence on flow parameters. However, accurate measurements of quantities of interest, such as shear stress, are difficult to make in vivo, thus numerical simulation becomes a valuable investigative tool.

The lattice Boltzmann model (LBM) $[13,14,15]$ uses a simplified kinetic equation to simulate fluid flow and has been applied to many general problems including turbulence [16, 17], magnetohydrodynamics [18] and multiphase flows [19].

The LBM has been applied to many of the problems involved in blood flow modelling. Artoli et al [20,21] modelled pulsatile blood flow in rigid walled models of the human aorta for two- and three dimensional geometries $[22,23]$. Krafczyk et al $[24,25]$ used a 3D LBM model to simulate the flow through a CarboMedics bileaflet heart valve at a range of fixed operating angels. Boyd et al has studied the flow characteristics in a two-dimensional carotid artery geometry with rigid walls [26, 27]. Fang and coworkers, along with other groups, have developed methods of modelling compliant walls in the context of blood flow modelling [28, 29, 30, 31, 32]. A number of groups have also used the LBM to study particle suspensions [33, 34, 35, 36, 37, 38, 39], stent development [40], prediction of coronary artery disease [41], blood clotting [36, 42] and vascular reconstruction $[43,44]$. The general usefulness, stability and versatility of the LBM for these applications is now well established [45, 46, 47, 48] and LBM blood flow 
modelling continues to be an active area of research.

In this paper the numerical model and the properties of the carotid artery simulation will be presented in sections 2 and 3 respectively. Analysis of the velocity in the artery will be presented in section 4 . Details of the shear in the body of the artery and at the wall will be presented in a companion paper [49].

\section{Theoretical Background}

The lattice Boltzmann method [13] has recently been developed as an alternative method for simulating a range of fluid flow using a modified molecular dynamics approach. In the LBM particle distribution functions, $f_{i}(\mathbf{x}, t)$ at point $\mathbf{x}$ at time $t$, are confined to move synchronously on a regular lattice. The distribution functions interact on the lattice in a way that conserves mass, momentum and ensures the fluid is isotropic and Galilean invariant. Here $i$ labels the lattice link the distribution function is on. The lattice used in this paper is the D3Q15, shown in figure 1.
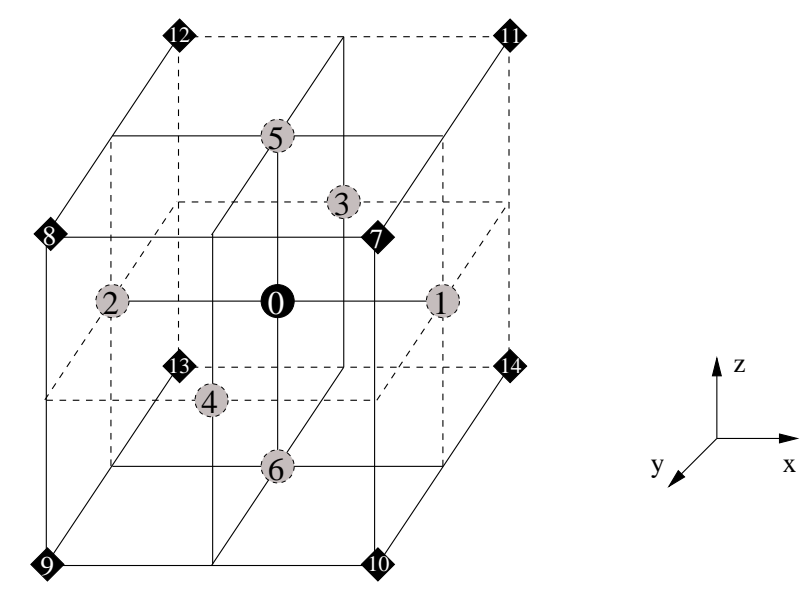

Figure 1. The D3Q15 lattice. The node is indicated by the black filled circle labelled 0 . Links $1-6$ are indicated by the gray filled circles. Links $7-14$ are indicated by the black diamonds.

The evolution of the distribution functions on the lattice is governed by the discrete Boltzmann equation $[13,50]$

$$
f_{i}\left(\mathbf{x}+\mathbf{e}_{i} \Delta x, t+\Delta t\right)=f_{i}(\mathbf{x}, t)+\Omega_{i}(\mathbf{x}, t), \quad(i=0,1 \cdots, 14),
$$

where for the D3Q15 lattice, see figure 1,

$$
\begin{array}{ll}
\mathbf{e}_{0} & =(0,0,0), \\
\mathbf{e}_{1,2}, \mathbf{e}_{3,4}, \mathbf{e}_{5,6} & = \pm(1,0,0), \pm(0,1,0), \pm(0,0,1), \text { and } \\
\mathbf{e}_{7,13}, \mathbf{e}_{8,14}, \mathbf{e}_{9,11}, \mathbf{e}_{10,12} & = \pm(1,1,1), \pm(-1,1,1), \pm(-1,-1,1), \pm(1,-1,1),
\end{array}
$$

and $\Omega_{i}$ is the collision operator. 
The fluid density $\rho$ and velocity $\mathbf{u}$ can be calculated directly from the distribution functions at each node by

$$
\rho=\sum_{i} f_{i} \text { and } \rho \mathbf{u}=\sum_{i} f_{i} \mathbf{e}_{i}
$$

respectively. It is assumed that the distribution functions $f_{i}$ can be expanded formally around a local equilibrium distribution such that

$$
f_{i}=f_{i}^{e q}+\varepsilon f_{i}^{n e q}
$$

where $\varepsilon$ is a small parameter often taken to be the Knudson number, $f_{i}^{e q}$ are equilibrium distribution function and $f_{i}^{n e q}$ are non-equilibrium distributions functions. $f_{i}^{e q}$ is selected such that

$$
\sum_{i} f_{i}^{e q}=\rho \text { and } \sum_{i} f_{i}^{e q} \mathbf{e}_{i}=\rho \mathbf{u}
$$

and it is assumed that the non-equilibrium distribution functions, $f_{i}^{n e q}$, can be further expanded as

$$
f_{i}^{n e q}=f_{i}^{(1)}+\varepsilon f_{i}^{(2)}+o\left(\varepsilon^{2}\right)
$$

where

$$
\sum_{i} f_{i}^{(k)}=\sum_{i} f_{i}^{(k)} \mathbf{e}_{i}=0, \quad k=1,2 .
$$

The collision operator $\Omega_{i}$ is given by the Bhatnagar-Gross-Krook approximation as $[13,50,51]$

$$
\Omega_{i}=\frac{-1}{\tau}\left[f_{i}(\mathbf{x}, t)-f_{i}^{e q}(\mathbf{x}, t)\right],
$$

where $\tau$ is the relaxation time and $f_{i}^{e q}(\mathbf{x}, t)$ is the equilibrium value of the distribution function, given by [50]

$$
f_{i}^{e q}(\mathbf{x}, t)=w_{i} \rho\left(1+3 \mathbf{e}_{i} \cdot \mathbf{u}+\frac{9}{2}\left(\mathbf{e}_{i} \cdot \mathbf{u}\right)^{2}-\frac{3}{2} \mathbf{u}^{2}\right),
$$

where

$$
\begin{array}{ll}
w_{0}=\frac{2}{9} & \\
w_{i}=\frac{1}{9} & \text { for } i=1-6, \text { and } \\
w_{i}=\frac{1}{72} & \text { for } i=7-14 .
\end{array}
$$

The relaxation time $\tau$ is related to the viscosity $\eta$ by

$$
\eta=\frac{(2 \tau-1) \rho}{6} .
$$



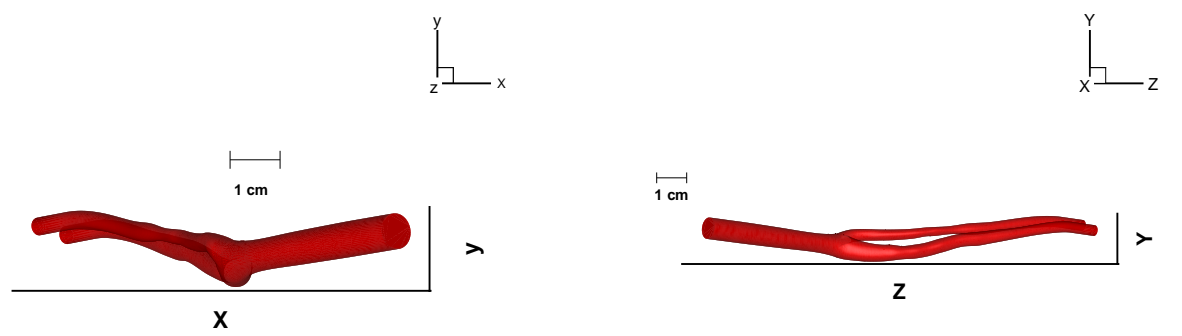

(a)

(b)

(c)

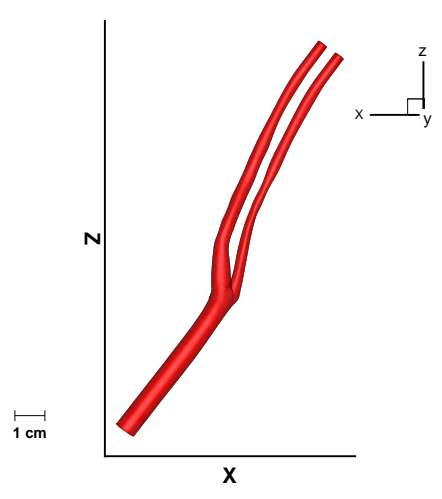

(d)

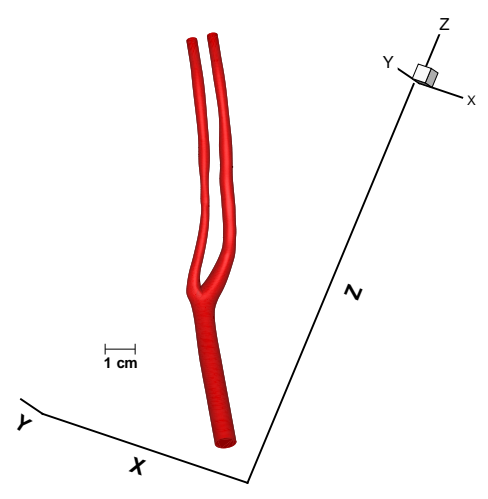

Figure 2. Carotid artery geometry shown in four different orientations, (a) $z$-axis projection, (b) $x$-axis projection, (c) $y$-axis projection and (d) geometry vertically oriented.

The LBM reproduces the Navier stokes equation in the nearly incompressible limit and is second order accurate in the body of the fluid [13]. The stress tensor for an incompressible fluid with pressure $p$ is given by

$$
\sigma_{\alpha \beta}=-p \delta_{\alpha \beta}+2 \eta S_{\alpha \beta},
$$

where $\delta_{\alpha \beta}$ is the Kronecker delta,

$$
S_{\alpha \beta}=\frac{1}{2}\left(\frac{\partial u_{\alpha}}{\partial x_{\beta}}+\frac{\partial u_{\beta}}{\partial x_{\alpha}}\right),
$$

is the strain rate tensor and $\alpha, \beta$ label the vector components: $\alpha, \beta=x, y$ or $z$. It can be shown [52] that $S_{\alpha \beta}$ can be calculated locally at each node in the LBM as

$$
S_{\alpha \beta}=-\frac{3}{2 \tau} \sum_{i} f_{i}^{(1)} e_{i \alpha} e_{i \beta} .
$$

The $f_{i}^{(1)}$ terms are usually calculated as part of the velocity calculations in the LBM algorithm. Thus calculating shear in this manner is efficient since it removes the need to calculate derivatives of the velocity. This method of calculating shear has been shown to maintain the second order accuracy of the LBM [53]. 
A sub-grid accurate extrapolation boundary scheme [54] is used here to implement the artery geometry in the model. This boundary scheme retains the second order nature of the LBM and is well suited to modelling artery geometries as it enables the shape of the artery wall to be simulated at a resolution greater than that provided by the underlying lattice $[26,27,55]$. In this scheme the shape of the geometry is defined by a series of boundary points $x_{b}$ which lie at the intersections of the geometry boundary and the lattice links, but not necessarily at a node of the D3Q15 lattice. These boundary points are characterised by the link direction they are on $(1-15)$ and the distance, $\Delta \in(0,1]$, from the nearest interior fluid node along that link. This information is used to create a fictitious distribution function that is streamed in from outside the geometry such that a no-slip boundary condition is imposed at $\mathbf{x}_{b}$.

\section{Methods}

Figures $2(\mathrm{a})$ - (d) and animation $1{ }^{* *}$ LINK $^{* *}$ show the 3D carotid artery geometry used in this paper. Figure 3 (a) shows the region of interest in this geometry for this paper, indicated by the black box. This region around the bifurcation of the carotid artery is known to be susceptible to atherosclerosis. This region is also chosen to be sufficiently far from the inlet and outlet boundaries so as to avoid boundary effects. Figure 3 (b) shows the plane slices that were taken through the bifurcation region for analysis, labelled $1-11$ from the bottom slice. These regions are spaced approximately 3.4 $\mathrm{mm}$ (24 lattice points) apart in the $z$ direction. The characteristics of the bifurcation's velocity will be analysed on these slices. The common carotid artery (CCA), internal carotid artery (ICA) and external carotid artery (ECA) are indicated by the arrows in both figures.

Simulations were performed in parallel on the 16 processor University of New England Beowulf cluster. The procedure used is detailed elsewhere [56]. A pulsatile waveform adapted from Holdsworth et al (1999) [57] is shown in figure 4.

This pulsatile waveform was implemented as a boundary condition at the base of the artery by setting the distribution functions at the entry equal to their equilibrium values, calculated from equation (9), for the desired profile and density. The profile was applied uniformly across the width of the entry except for a boundary layer region of width approximately $1 \mathrm{~mm}$ over which the velocity was linearly reduced to zero at the wall of the artery.

The unknown distribution functions at an exit site $\mathbf{x}_{E}$ were found from a linear extrapolation, based on [58]:

$$
f_{i}\left(\mathbf{x}_{E}, t+1\right)=2 f_{i}\left(\mathbf{x}_{E}+\mathbf{e}_{i}, t+1\right)-f_{i}\left(\mathbf{x}_{E}+2 \mathbf{e}_{i}, t+1\right) .
$$

Times of interest in the pulse cycle are indicated by the crosses in figure 4 . The times selected for analysis, including local maxima and minima of the velocity waveform, were $t=0.03 \mathrm{~s}, t=0.1 \mathrm{~s}, t=0.17 \mathrm{~s}, t=0.24 \mathrm{~s}, t=0.31 \mathrm{~s}$ and $t=0.6 \mathrm{~s}$. The velocity characteristics in the artery will be analysed in more detail at these times. 
(a)
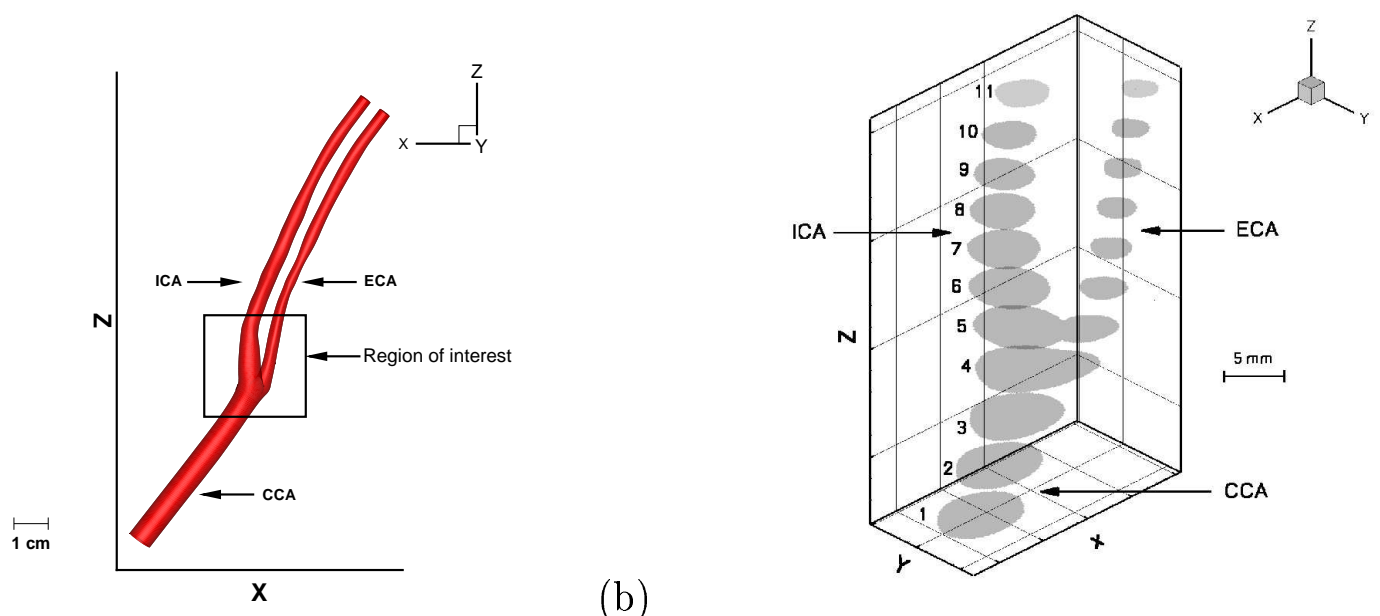

Figure 3. (a) Carotid artery geometry with region of interest around the bifurcation indicated, (b) Plane slices through the bifurcation, labelled 1 - 11. Slices are spaced approximately $3.4 \mathrm{~mm}$ apart in the $Y$ direction. In both images the common carotid artery (CCA), internal carotid artery (ICA) and external carotid artery (ECA) are indicated.

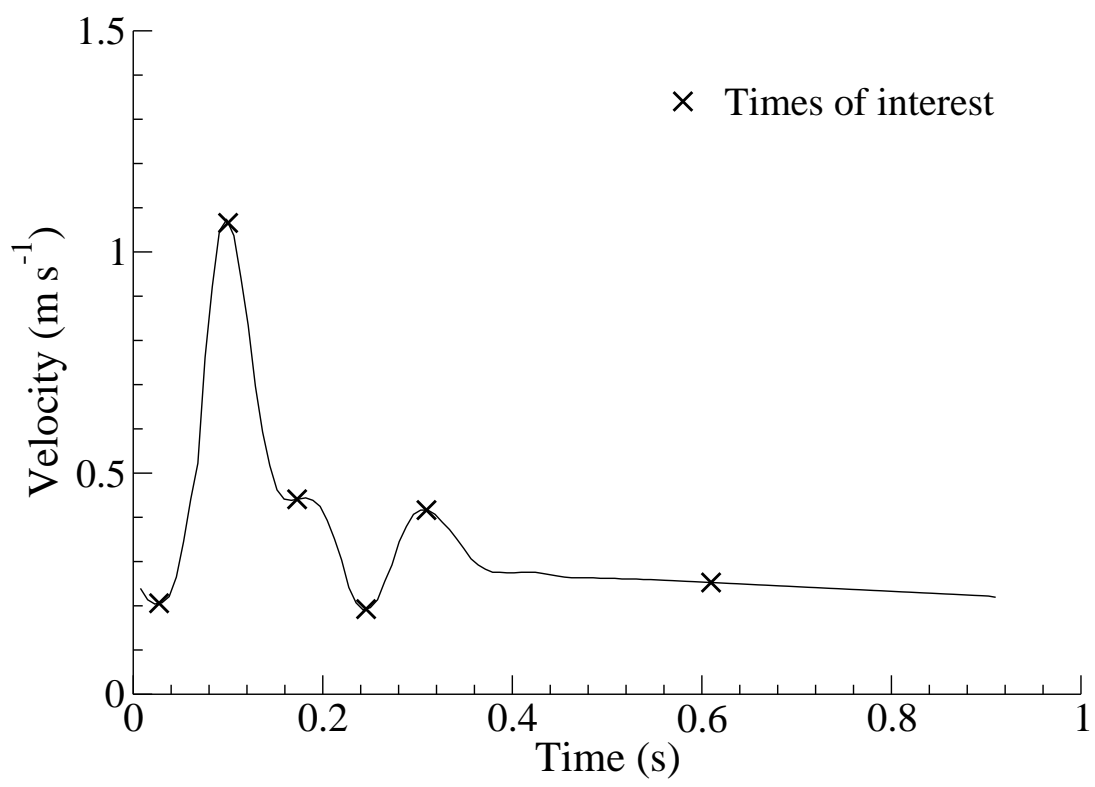

Figure 4. Pulse waveform adapted from [57], with times of interest indicated. Times from left to right are $t=0.03 \mathrm{~s}, t=0.1 \mathrm{~s}, t=0.17 \mathrm{~s}, t=0.24 \mathrm{~s}, t=0.31 \mathrm{~s}$ and $t=0.6$ s.

The Reynolds number $\left(R e_{\delta}\right)$, based on the boundary layer thickness $\delta$, and Womersley $(\alpha)$ dimensionless parameters for this type of flow are defined in the following 
Table 1. A comparison of artery simulation parameters and physiological parameters obtained from Holdsworth et al (1999) [57].

\begin{tabular}{ccc}
\hline Parameter & Holdsworth et al (1999) [57] & 3D geometry (Lattice units) \\
\hline$u_{0}$ & $1.07 \mathrm{~m} \mathrm{~s}^{-1}$ & 0.03 \\
$L$ (base of the CCA) & $6.4 \times 10^{-3} \mathrm{~m}$ & 45 \\
Total number of nodes & - & $1.5 \times 10^{6}$ \\
$\eta$ & $3.5 \times 10^{-6} \mathrm{~Pa} \mathrm{~s}$ & $6.899 \times 10^{-4}$ \\
$T$ & $0.91 \mathrm{~s}$ & 228200 \\
$R e$ & 200 & 200 \\
$\alpha$ & 4.5 & 4.5 \\
\hline
\end{tabular}

manner,

$$
\begin{aligned}
R e_{\delta} & =u_{0} \sqrt{\frac{T}{\nu \pi}}, \text { and } \\
\alpha & =L \sqrt{\frac{\pi}{2 T \nu}},
\end{aligned}
$$

where $u_{0}$ is the peak artery velocity, $\nu$ is the kinematic viscosity, $T$ is the period of the first harmonic of the waveform, corresponding to the pulse period, and $L$ is the artery diameter. The human carotid artery typically has values of around $R e_{\delta}=200$ and $\alpha=4.5$ for these dimensionless parameters.

The parameters used in the simulation are given in table 1. Blood was assumed to be Newtonian, as this assumption has been found to be appropriate for carotid artery blood flow [27].

The simulations were run for four pulse periods, with the data output every $100^{\text {th }}$ of a period during the last period. It was found that small variations in the entry parameters, such as the width of the boundary layer, did not make a significant difference to the presented results.

\section{Results}

Figures 5 (a) - (f) show the velocity magnitudes at the times indicated in figure 4 . The velocities observed in the $3 \mathrm{D}$ carotid artery bifurcation agree well with physiological data $[6,11,8]$, as well as with previous $2 \mathrm{D}$ simulation results [26, 27]. The velocity magnitude over the whole pulse is presented in animation $2 * * \mathrm{LINK}^{* *}$.

A number of interesting features are observed. The highest velocity flow in the CCA is in the central region of the artery during the whole pulse cycle. The cross sectional velocity profiles are approximately parabolic in slices $1-4$, and also have approximately the same peak velocities for times $t=0.03 \mathrm{~s}, t=0.1 \mathrm{~s}$ and $t=0.17 \mathrm{~s}$, figure 5 (a) - (c). A reduction in the velocity occurs when the CCA widens into the bifurcation region. 
(a)

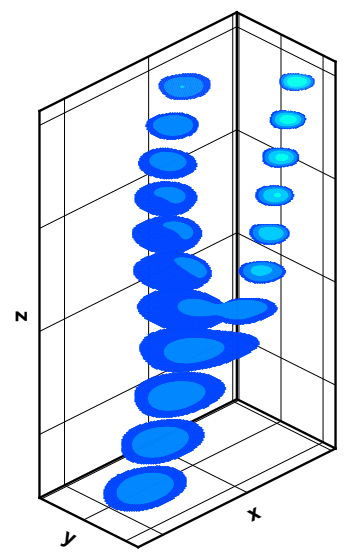

Velocity $\left(\mathrm{m} \mathrm{s}^{-1}\right)$

0.7
0.65
0.6

\begin{tabular}{|l|}
0.65 \\
0.6 \\
0.55 \\
0.5 \\
0.45 \\
0.4
\end{tabular}

0.35

0.3
0.25

$\begin{array}{ll} & 0.25 \\ & 0.2 \\ 0.15\end{array}$

0.15
0.1
0.05

0.05
0

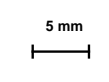

(c)

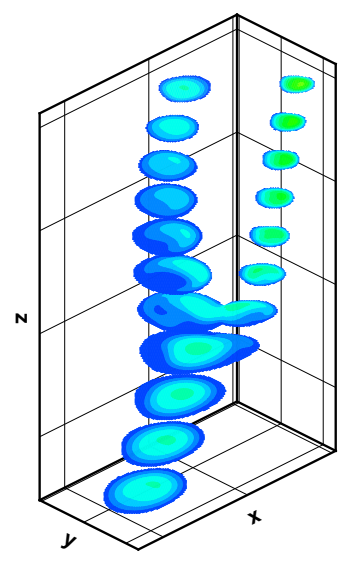

Velocity $\left(\mathrm{m} \mathrm{s}^{-1}\right)$

0.7
0.65
0.6

0.65
0.65
0.5
0.45

\begin{tabular}{ll}
0.45 \\
0.4 \\
0.35 \\
\hline
\end{tabular}

0.3
0.25

0.25
0.2
0.15

0.1
0.05

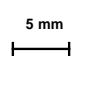

(e)

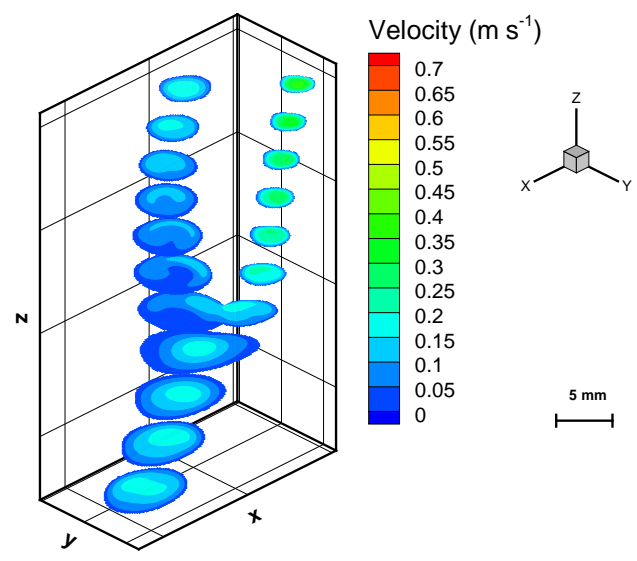

(f)

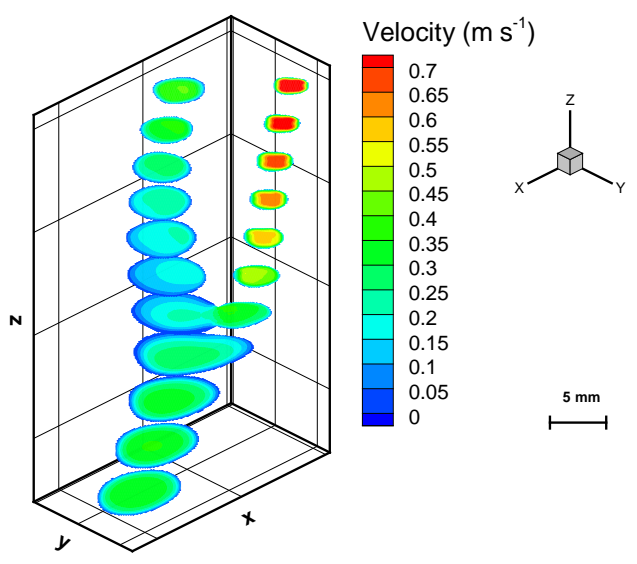

(b)

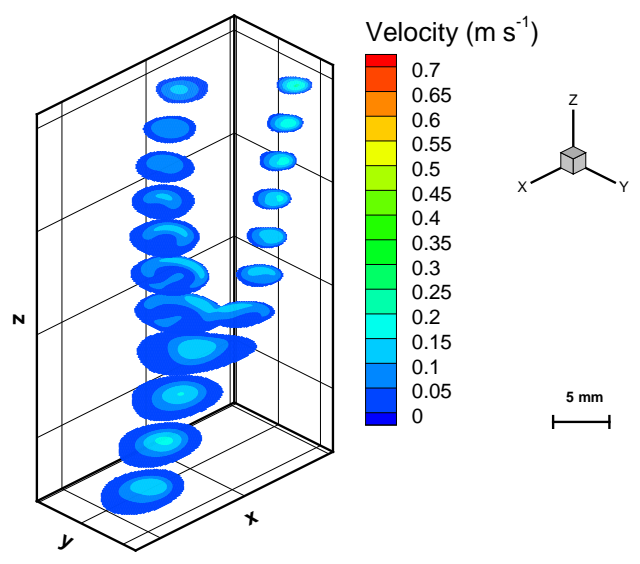

(d)

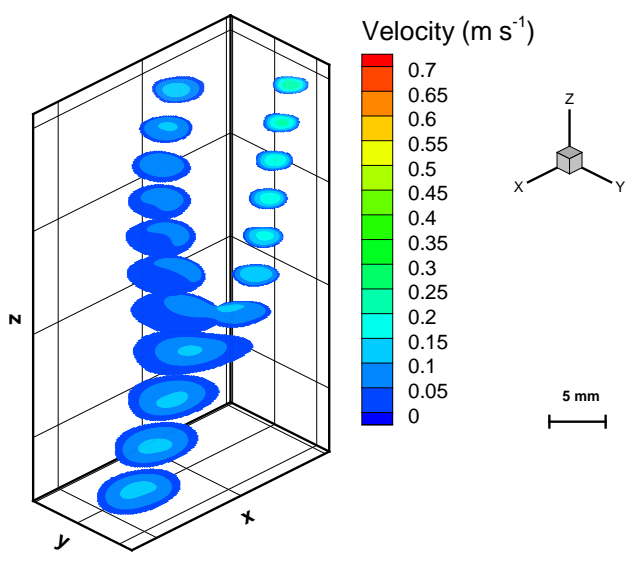

Figure 5. Velocity magnitude in the carotid artery at times (a) $t=0.03 \mathrm{~s}$, (b) $t=0.10$ $\mathrm{s},(\mathrm{c}) t=0.17 \mathrm{~s}$, (d) $t=0.24 \mathrm{~s}$, (e) $t=0.31 \mathrm{~s}$, (f) $t=0.60 \mathrm{~s}$. 
The branching in the CCA forces the blood flow to divide between the ECA and ICA. Low velocity regions appear in the lower part of the ICA, on the outer wall of the bifurcation. This low velocity region extends about halfway up the region of the ICA shown, and exists for the whole pulse cycle. The flow profiles in this region are not parabolic and exhibit higher velocities closer to the inner wall of the ICA. Parabolic like flow is restored further up the ICA. These flow characteristics agree well with the literature reports of the flow characteristics in the carotid bifurcation as well as with previously reported results $[6,11,26,8]$.

The blood velocity in the ECA is relatively higher than that seen in the other regions of the artery, due to its smaller diameter. The highest velocity flow of $0.85 \mathrm{~m} \mathrm{~s}^{-1}$ is seen in the upper regions of the ECA. The velocity profiles in this region are approximately parabolic for most of the pulse period, except at the base of the ECA.

Regions of low near wall velocity are also seen at the outer wall of the ECA at its base. Once again the presence of this region of low velocity flow agrees well with the characteristics reported in the literature and with previous $2 \mathrm{D}$ results $[6,11,26,8]$. The velocity at the base of the ECA is in general higher than that seen at the base of the ICA. Movement in the position of the peak velocity in this region of the artery is also seen, although to a lesser extent than that seen in the ICA. This effect is confined to the region close to the bifurcation.

Evidence of flow circulation is observed in the lower part of the ICA, figures 5 (c) (e). In order to examine this effect in greater detail the $u_{z}$ velocity component on slices $5-7$, see figure 3 (b), were examined at the times indicated in figure 4 . Animation 3 ${ }^{* *}$ LINK $^{* *}$ presents these results for the whole pulse period. The portion of the artery considered here is approximately aligned with the z-axis, see figure 2 . The results are shown in figures 6 (a) - (f).

Flow circulation in the ICA can be seen at times $t=0.17-0.31 \mathrm{~s}$, figures 6 (c) - (e) respectively. These times correspond to the dip in the artery pulse, see figure 4. Retrograde flow is seen near the outer wall of the bifurcation and even extends into the central artery region for part of the pulse period, however the magnitude of the negative velocity is relatively small compared to the positive velocity seen near the inner wall.

In addition, movement in the position of the peak velocity is seen in the the central regions of the CCA and ICA, see figures 5 and 6 and animation $2^{* *}$ LINK $^{* *}$. In the CCA the peak velocity of the flow perambulates around the center of the artery. More significant movements in the peak position can be seen in the ICA. At time $t=0.10$, corresponding to the peak of the pulsatile waveform, we observe that the peak velocity is around the center of the ICA branch; however at times of lower inflow velocity the peak is located at various points close to the outer wall. These flow properties depict a more complicated flow situation than that seen in a previous 2D model [26, 27]. 
(a)

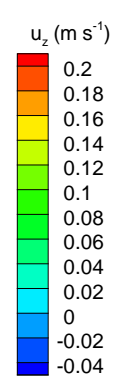

(c)

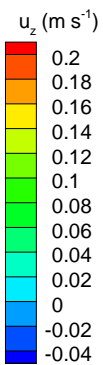

(e)

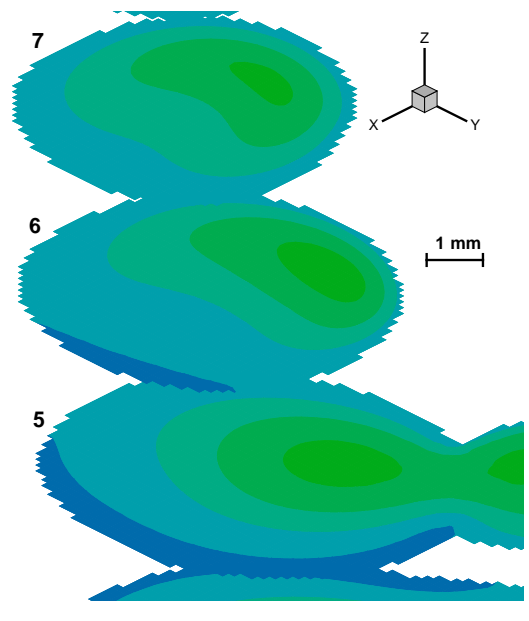

(b)
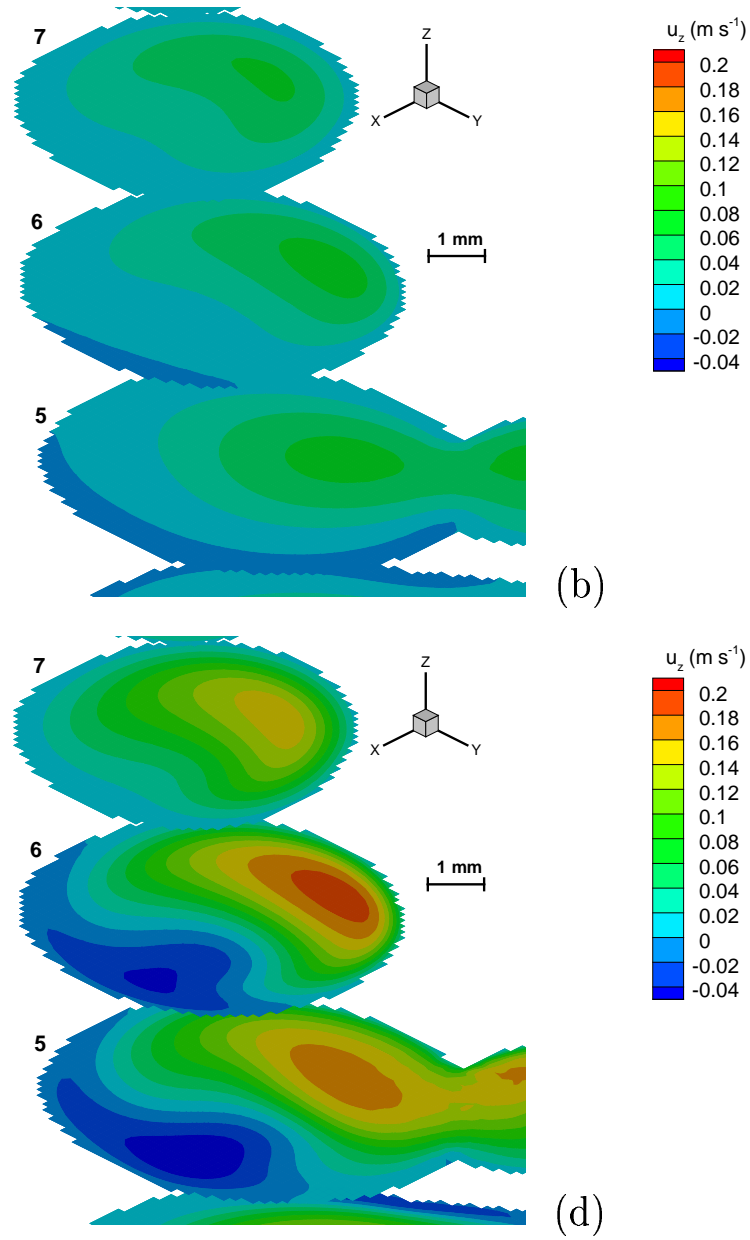

(d)
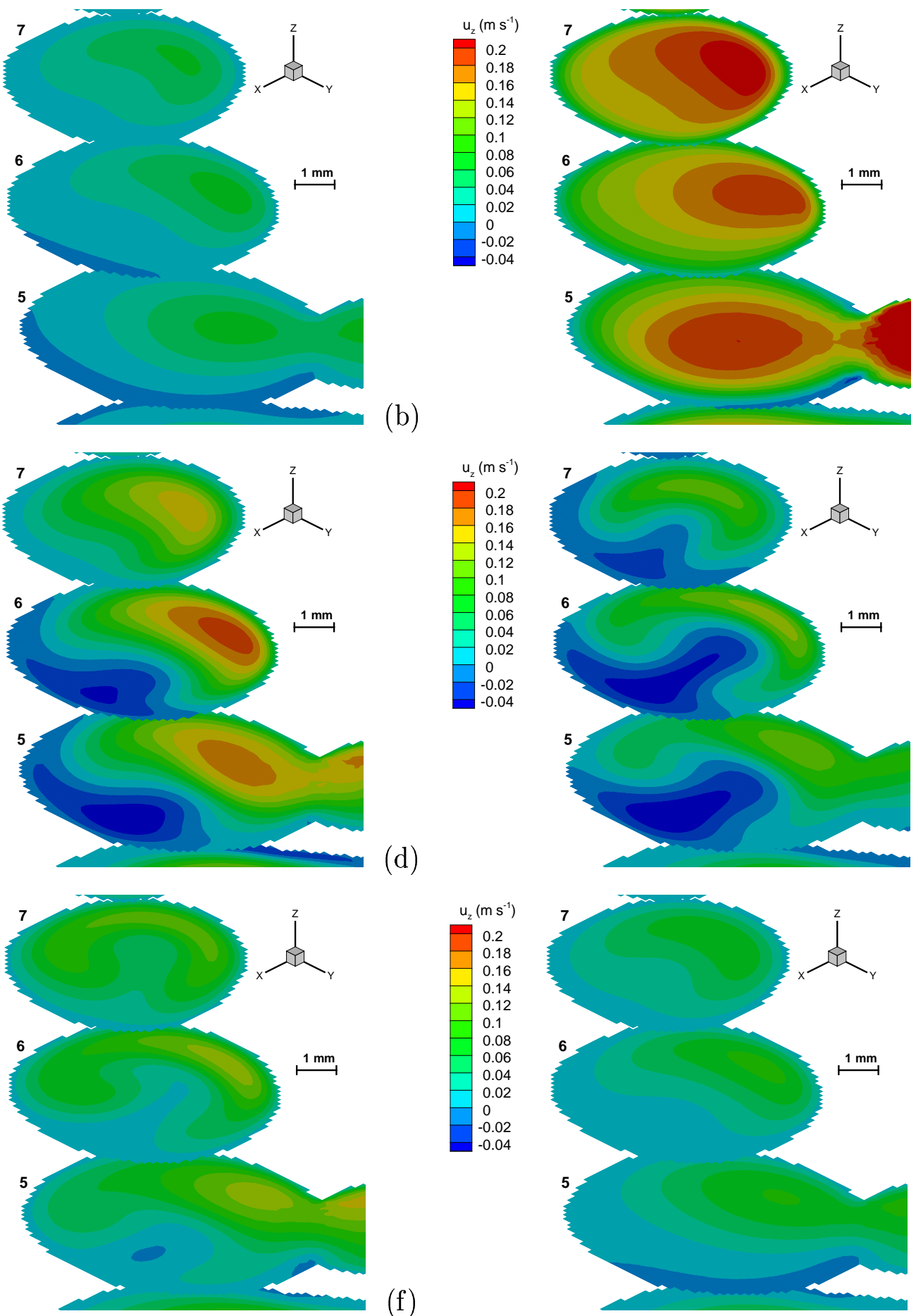

(f)
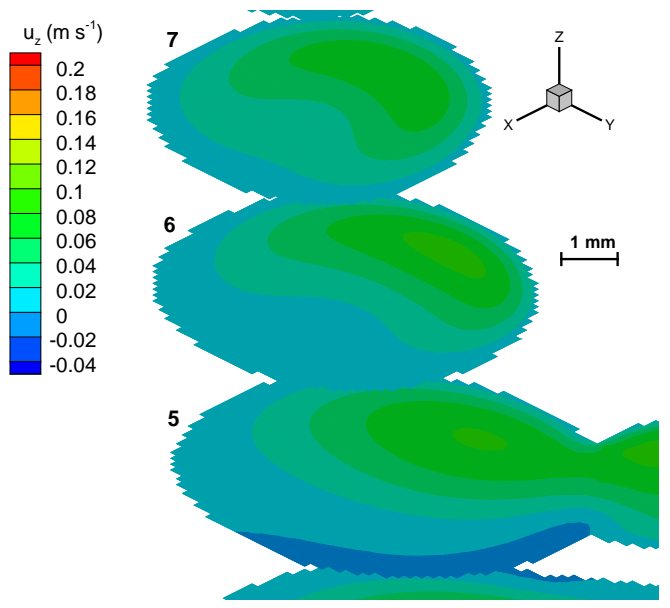

Figure 6. $u_{z}$ velocity component in the carotid artery at times (a) $t=0.03 \mathrm{~s}$, (b) $t=0.10 \mathrm{~s}$, (c) $t=0.17 \mathrm{~s}$, (d) $t=0.24 \mathrm{~s}$, (e) $t=0.31 \mathrm{~s}$, (f) $t=0.60 \mathrm{~s}$. 


\section{Conclusion}

The 3D carotid artery model has been used to study the properties of the velocity over a pulse cycle of Newtonian blood flow. The velocity characteristics presented here agree well with literature reports of flow in the human carotid artery as well as with previous $2 \mathrm{D}$ results. Physiologically accurate features are observed, including a slow down of flow in the bifurcation region, low near wall velocities on the outer walls of the ECA and ICA and circulating flows $[6,11,8]$. The 3D model exhibits the same general features as those observed in previous 2D models; however more complex behaviour, such as the spatial movement of the velocity peak, is only observable in the $3 \mathrm{D}$ case.

The LBM has been shown to lend itself to simulating arterial blood flow. Analysis of the shear field in the artery and at the walls will be presented in a companion paper [49] where the combined properties of velocity and shear will be considered at relevant positions in the carotid artery with respect to the progression of the atherosclerosis.

\section{Acknowledgments}

The authors would like to acknowledge the technical assistance of N. Gaywood, A. Saint and S. Ryan. This work was also partially supported by Sigma Xi Grant no. 10040015 and the Australian Postgraduate Award (APA), this assistance is gratefully acknowledged.

\section{References}

[1] Silverthorn D U. Human Physiology: An Integrated Approach, Second Edition. Pearson Education International, 2001.

[2] Caro C G. Vascular fluid dynamics and vascular biology and disease. Math. Meth. Appl. Sci., 24:1311-1324, 2001.

[3] Murray C J L and Lopez A D. The global burden of disease: a comprehensive assessment of mortality and disability from diseases, injuries, and risk factors in 1990 and projected to 2020. Harvard school of public health MA, 1996.

[4] Stamler J. Arteriosclerosis. In D. I. Abramson, editor, Blood vessels and lymphatics. Academic Press, 1962.

[5] Ross R and Glomset J A. The pathogenesis of atherosclerosis(I). N. Engl. J. Med., 295:369-377, 1976.

[6] Malek A M, Alper S L, and Izumo S. Hemodynamic Shear Stress and Its Role in Atherosclerosis. J. Amer. Med. Assoc., 282:2035-2042, 1999.

[7] Asakura T and Karino T. Flow Patterns and Spatial Distribution of Atherosclerotic Lesions in Human Coronary Arteries. Circ. Res., 66:1045-1066, 1990.

[8] Quarteroni A, Tuveri M, and Veneziani A. Computational vascular fluid dynamics: problems, models and methods. Comput. Visual. Sci., 2:163-197, 2000.

[9] Caro C G, Fitz-Gerald J M, and Schroter R C. Atheroma and arterial wall shear, Observation, correlation and proposal of a shear dependant mass transfer mechanism for atherogenesis. Proc. R. Soc. London B, 177:109-159, 1971.

[10] Zarins C K, Giddens D P, Bharadvaj B K, Sottiurai V S, Mabon R F, and Glagov S. Carotid Bifurcation Atherosclerosis, Quantitative Correlation of Plaque Localisation with Flow Velocity Profiles and Wall Shear Stress. Circ. Res., 53:502-514, 1983. 
[11] Gnasso A, Irace C, Carallo C, De Franceschi M S, Motti C, Mattioli P L, and Pujia A. In Vivo Association Between Low Wall Shear Stress and Plaque in Subjects With Asymmetrical Carotid Atherosclerosis. Stroke, 28:993-998, 1997.

[12] Solberg L A and Eggen D A. Localization and Sequence of Development of Atherosclerotic Lesions in the Carotid and Vertebral arteries. Circ., 43:711-724, 1971

[13] Chen S and Doolen G D. Lattice boltzmann method for fluid flows. Ann. Rev. Fluid Mech., 30:329-364, 1998.

[14] Succi S. The Lattice Boltzmann Equation for Fluid Dynamics and Beyond. Oxford University Press, 2001.

[15] Wolf-Gladrow D A. Lattice-Gas Cellular Automata and Lattice Boltzmann Models, An introduction. Springer, 2000.

[16] J. A. Cosgrove, J. M. Buick, S. J. Tonge, C. G. Munro, C. A. Greated, and D. M. Campbell. Application of the lattice Boltzmann method to transition in oscillatory channel flow. J. Phys. A, 36(10):2609-2620, 2003.

[17] J. A. Cosgrove, J. M. Buick, and S. J. Tonge. Evolution of turbulence in an oscillatory flow in a smooth-walled channel: A viscous secondary instability mechanism. Phys. Rev. E, 68(2):026302, 2003.

[18] Chen S, Chen H, Martinez D, and Matthaeus W. Lattice Boltzmann model for simulation of magnetohydrodynamics. Phys. Rev. Lett., 67(27):3776-3779, 1991.

[19] Shan $\mathrm{X}$ and Chen H. Lattice Boltzmann model for simulating flows with multiple phases and components. Phys. Rev. E, 47:1815-1819, 1993.

[20] Artoli A M, Hoekstra A G, and Sloot P M A. Simulation of a systolic cycle in a realistic artery with the Lattice Boltzmann BGK method. Int.J. Mod. Phys. B, 17(1-2):95-98, 2003.

[21] Artoli A M, Hoekstra A G, and Sloot P M A. Mesoscopic simulations of systolic flow in the human abdominal aorta. J. Biomech., 39(5):873-884, 2006.

[22] Artoli A M, Hoekstra A G, and Sloot P M A. Accuracy of 2D Pulsatile Flow in the Lattice Boltzmann BGK Method. In Sloot P M A, Kenneth Tan C J, Dongerra J J, and Hoekstra A G, editors, Proceedings of the International Conference in Computational Science (ICCS) 2002, pages 361-370, Amsterdam, Holland, 2002.

[23] Artoli A M, Hoekstra A G, and Sloot P M A. 3D Pulsatile Flow With the Lattice Boltzmann BGK Method. Int.J. Mod. Phy. C, 13(8):1119-1134, 2002.

[24] Krafczyk M, Cerrolaza M, Schulz M, and Rank E. Analysis of 3D transient blood flow passing through and artificial aortic valve by Lattice-Boltzmann methods. J. Biomech., 31:453-462, 1998.

[25] Krafczyk M, Tölke J, Rank E, and Schulz M. Two-dimensional simulation of the fluid-structure interaction using Lattice-Boltzmann methods. Comp. Struct., 79:2031-2037, 2001.

[26] Boyd J, Buick J M, Cosgrove J A, and Stansell P. Application of the lattice Boltzmann model to simulated stenosis growth in a two-dimensional carotid artery. Phys. Med. Biol., 50:4783-4796, 2005 .

[27] Boyd J and Buick J M. Comparison of Newtonian and non-Newtonian flows in a two-dimensional carotid artery model using the lattice Boltzmann method. Phys. Med. Biol., 52(20):6215-6228, 2007.

[28] Fang H, Wang Z, Lin Z, and Liu M. Lattice Boltzmann simulation of viscous fluid systems with elastic boundaries. Phys. Rev. E, 57(1):R25-R28, 1998.

[29] Fang H, Wang Z, Lin Z, and Liu M. Lattice Boltzmann method for simulating the viscous flow in large distensible blood vessels. Phys. Rev. E, 65:051925:1-11, 2002.

[30] Lu X Y, Yi H H, Chen J Y, and Fang H P. Lattice BGK Simulations of the Blood Flow in Elastic Vessels. Chinese Phys. Lett., 23:738-741, 2006.

[31] Hoekstra A G, van't Hoff J, Artoli A M, and Sloot P M A. Unsteady flow in a 2D elastic tube with the LBGK method. Future Gener. Comp. Sy., 20:917-924, 2004.

[32] Leitner D, Wassertheurer S, Hessinger M, and Holzinger A. A Lattice Boltzmann Model for 
pulsatile blood flow in elastic vessels. e $\mathscr{G} i$ Elektrotechnik und Informationstechnik, 123(4):152$155,2006$.

[33] Ladd A J C and Verberg R. Lattice Boltzmann simulations of particle-fluid suspensions. J. Stat. Phys., 104:1191-1251, 2001.

[34] Migliorini C, Quin Y-H, Chen H, Brown E B, Jain R K, and Munn L L. Red Blood Cells Augment Leukocyte Rolling in a Virtual Blood Vessel. Biophys. J., 83:1834-1841, 2002.

[35] Sun C and Munn L L. Particulate Nature of Blood Determines Macroscopic Rheology: A 2-D Lattice Boltzmann Analysis. Biophys. J., 88:1635-1645, 2005.

[36] Ouared R and Chopard B. Lattice Boltzmann Simulations of Blood flow: Non-Newtonian Rheology and Clotting Processes. J. Stat. Phys., 121(1/2):209-221, 2005.

[37] Li H B, Fang H, Lin Z, Xu S X, and Chen S. Lattice Boltzmann simulation on particle suspensions in a two-dimensional symmetric stenotic artery. Phys. Rev. E, 69(3):031919, 2004.

[38] Li H B, Lu X Y, Fang H P, and Lin Z F. Simulation of multi-particle suspensions in a quasi-twodimensional symmetric stenotic artery with Lattice Boltzmann method. PCFD, 5(1-2):65-74, 2005.

[39] Li H B, Yi H H, Shan X W, and Fang H P. Shape changes and motion of a vesicle in a fluid using a lattice Boltzmann model. Euro. Phys. Lett., 81:54002-1-5, 2008.

[40] Chen C, Chen H, Freed D, Shock R, Staroselsky I, Zhang R, Ümit Coşkun A, Stone P H, and Feldman C L. Simulation of blood flow using extended Boltzmann kinetic approach. Physica A, 362(1):174-181, 2006.

[41] Ümit Coskun A, Chen C, Stone P H, and Feldman C L. Computational fluid dynamics tools can be used to predict the progression of coronary artery disease. Physica A, 362(1):182-190, 2005.

[42] Bernsdorf J, Harrison S E, Smith S M, Lawford P V, and Hose D R. Concurrent numerical simulation of flow and blood clotting using the lattice Boltzmann technique. IJBRA, 2(4):371$380,2006$.

[43] Sloot P M A, Tirado-Ramos A, Hoekstra A G, and Bubak M. An interactive grid for non-invasive vascular reconstruction. In IEE International Symposium on Cluster and Grid Computing 2004 (CCGrid 2004), pages 309-319, 2004.

[44] Abrahamyan L, Schaap J A, Hoekstra A G, Shamonin D, Box F M A, van der Feest R J, Reiber $\mathrm{J} \mathrm{H} \mathrm{C}$, and Sloot P M A. A problem solving environment for image-based computational hemodynamics. In Lecture notes in Computer Science - Volume 3514. Springer, 2005.

[45] Axner L, Hoekstra A G, and Sloot P M A. Simulating time harmoic flows with the lattice Boltzmann method. Phys. Rev. E, 75:036709:1-7, 2007.

[46] Kang X-Y, Liu D-H, Zhou J, and Jin Y-J. Simulating High Reynolds Number Flow by Lattice Boltzmann Method. Chinese Phys. Lett., 22:1456-1459, 2005.

[47] Hoekstra A G. Image-based computational hemodynamics with the lattice boltzmann method. In K. J. Bathe, editor, Computational Fluid and Solid Mechanics 2005, pages 672-675. Elsevier, 2005.

[48] Yi H H, Xu S X, Qian Y H, and Fang H P. Lattice Boltzmann simulation of blood flow in blood vessels with the rolling massage. Chinese Phys. Lett., 22(12):3210-3213, 2005.

[49] J Boyd and J M Buick. Three-dimensional modelling of the human carotid artery using the lattice Boltzmann method. II: Shear analysis. submitted.

[50] Qian Y H, d'Humieres D, and Lallemand P. Lattice BGK models for Navier-Stokes equation. Europhys. Lett., 17(6):479-484, 1992.

[51] Bhatnagar P L, Gross E P, and Krook M. A model for collision processes in gases. I: small amplitude processes in charged and neutral one-component system. Phys. Rev., 94:511-525, 1954.

[52] Artoli A M. Mesoscopic Computational Haemodynamics. Ponsen \& Looijen, Wageningen, 2003.

[53] Boyd J, Buick J M, and Green S. A second order accurate lattice Boltzmann non-Newtonian flow model. J. Phys. A-Math. Gen., 39:14241-14247, 2006.

[54] Guo Z, Zheng C, and Shi B. An extrapolation method for boundary conditions in lattice Boltzmann 
method. Phys. Fluids, 14(6):2007-2010, 2002.

[55] Boyd J, Buick J M, Cosgrove J A, and Stansell P. Application of the lattice Boltzmann method to arterial flow simulation: Investigation of boundary conditions for complex arterial geometries. Australas. Phys. Eng. Sci. Med., 27(4):207-212, 2004.

[56] Boyd J, Ryan S J, and Buick J M. A parallel Lattice Boltzmann Model of a Carotid Artery. American Institute of Physics Conference Proceedings, 2008. In press.

[57] Holdsworth D W, Norley C J D, Frayne R, Steinman D A, and Rutt B K. Characterization of common carotid artery blood-flow waveforms in normal human subjects. Physiol. Meas., 20:219-240, 1999.

[58] Neal M. A study of the brass intrument lip reed mechanism using artificial lips and lattice Boltzmann flow simulation. PhD thesis, The University of Edinburgh, 2002. 